\title{
The Effect of Low-Power Laser Therapy on the TGF/ $\beta$ Signaling Pathway in Chronic Kidney Disease: A Review
}

\author{
Behnaz Ahrabi', Maryam Bahrami², Reza Moghadasali ${ }^{3}$,Mona Zamanian-Azodi', Maryam Sadat Khoramgah', \\ Fatemeh Sadat Tabatabaei Mirakabad ${ }^{1}$, Shahram Darabi ${ }^{5}$, Hojjat Allah Abbaszadeh ${ }^{1 *}$ \\ ${ }^{1}$ Laser Application in Medical Sciences Research Center, Shahid Beheshti University of Medical Sciences, Tehran, Iran \\ ${ }^{2}$ Department of Biology and Anatomical Sciences, school of medicine, Shahid Beheshti University of Medical Sciences, \\ Tehran, Iran \\ ${ }^{3}$ Department of Stem Cells and Developmental Biology, Cell Science Research Center, Royan Institute for Stem Cell Biology \\ and Technology, ACECR, Tehran, Iran. \\ ${ }^{4}$ Proteomics Research Center, Shahid Beheshti University of Medical Sciences, Tehran, Iran \\ ${ }^{5}$ Cellular and Molecular Research Center, Qazvin University of Medical Science, Qazvin, Iran
}

\author{
*Correspondence to \\ Hojjat-Allah Abbaszadeh. \\ Laser Application in Medical \\ Sciences Research Center \\ and Department of Biology \\ and Anatomical Sciences, \\ school of medicine, Shahid \\ Beheshti University of Medical \\ Sciences, Tehran, Iran. \\ P.O.Box: $19395-4719$. \\ Tel:+98-21-23872555; \\ Fax: +98-21-22439976; \\ Email: \\ Dr.abbaszadeh79@gmail.com, \\ Dr.abbaszadeh@sbmu.ac.ir \\ Published online March 15, \\ 2020

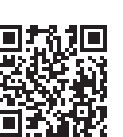

\begin{abstract}
Objective: The purpose of this study is to investigate the effects of low-power lasers on kidney disease by investigating several studies.

Methods: A number of articles from 1998 to 2019 were chosen from the sources of PubMed, Scopus, and only the articles studying the effect of low-power lasers on kidney disease were investigated.

Results: After reviewing the literature, 21 articles examining only the effects of low-power lasers on kidney disease were found. The results of these studies showed that the parameter of the lowpower laser would result in different outcomes. So, a low-power laser with various parameters can be effective in the treatment of kidney diseases such as acute kidney disease, diabetes, glomerulonephritis, nephrectomy, metabolic syndrome, and kidney fibrosis. Most studies have shown that low-power lasers can affect TGF $\beta 1$ signaling which is the most important signaling in the treatment of renal fibrosis.

Conclusion: Lasers can be effective in reducing or enhancing inflammatory responses, reducing fibrosis factors, and decreasing reactive oxygen species (ROS) levels in kidney disease and glomerular cell proliferation.

Keywords: Low-power laser therapy; Chronic kidney disease; TGF $\beta 1$ signaling.
\end{abstract}

\section{Introduction}

The kidney is an organ with high blood flow. It has a functional unit called the nephron which consists of structural and functional parts, including glomeruli and tubules. ${ }^{1}$ Chronic kidney disease (CKD) is a progressive disease in which the function of the kidney is impaired, leading to an increase in urea and creatinine levels within the blood, an increase in blood pressure, acidosis, hyperkalemia, a decrease in the calcium level and kidney filtration, and also kidney fibrosis. ${ }^{2-4}$ The progression of the disease can lead to interstitial nephritis, glomeruli, and fibrosis. ${ }^{5}$ The prevalence of the disease in the world is $10 \%$. If it is left untreated, it can lead to end-stage renal disease which would be costly due to possible further kidney transplantation or dialysis. ${ }^{6}$ In more than 100 countries worldwide, people are not able to receive longterm medical treatment. Thus, we will see annually more than one million deaths worldwide due to the untreated disease. $^{7}$ By 1990, kidney disease was ranked 27th in the list of causes of the total number of deaths worldwide, reaching 18 th by $2010 .{ }^{7}$ In fact, $25 \%$ of people whose ages range from 65 to 74 and who are diagnosed with CKD are men and $20 \%$ of them are also women. CKD result from other diseases such as diabetes, obesity, atherosclerosis, and high blood pressure. ${ }^{8}$ Fibrosis is also attributed to the overgrowth and scar of various tissues as well as to the presence of extracellular elements including collagen, mainly due to mesenchymal cells residing in the tissue. In fact, fibrosis is the result of chronic inflammatory reactions. The etiology of fibrosis is not completely clear. Studies have shown that kidney fibrosis occurs in four stages. During the first stage, kidney tissue could be damaged due to diabetes, inflammation, and such diseases as glomerulonephritis and sclerosis. Diabetes

Please cite this article as follows: Ahrabi B, Bahrami M, Moghadasali R, Zamanian-Azodi M, Khoramgah MS, Tabatabaei Mirakabad FS. The effect of low-power laser therapy on the TGF/ $\beta$ signaling pathway in chronic kidney disease: a review. J Lasers Med Sci. 2020;11(2):220225. doi:10.34172/jlms.2020.36. 
and hypertension play a major role. ${ }^{1}$ In the second stage, inflammatory cells, including mast cells, dendritic cells, lymphocytes, neutrophils, and macrophages, secrete fibrotic and inflammatory cytokines (IL6, IL1 and tumor necrosis factor-alpha). ${ }^{9}$ The healing process of damaged tissue is categorized into two distinct phases: (1) The healing phase in which damaged cells are replaced by the same type of cell and (2) the second phase which is known as fibrosis in which the connective tissue is replaced with normal tissue. Although the second phase seems beneficial at the beginning, in case of an unregulated process, the pathogenic process could be initiated. ${ }^{10}$ At stage III, many of the factors involved in kidney pathology, including CTGF, NF- $\kappa \beta$, TGF- $\beta 1$, and PAI1 increase following the TGF- $\beta$ signaling pathway when the inflammation pathway is not properly controlled, which induces MKK3- P38, MKK4-JNK signaling pathway through Smad, or non-Smad signaling pathways. Afterward, the kidney cells continuously synthesize extracellular matrix elements such as collagen type I and fibronectin in the glomeruli, kidney tubules, and kidney vessels, so that in the fourth stage these cells can be involved in the process of fibrosis. Epithelial cells, endothelial cells, inflammatory cells, fibroblast cells, pericytes, and myofibroblasts can be implicated. ${ }^{11}$ The pericytes and epithelial cells in the kidney may undergo the EMT process (conversion of epithelial cells to mesenchymal cells) to become myofibroblasts. An active form of myofibroblasts is an important cell mediator in fibrosis. They are known as the primary collagen-producing cells that could result in fibrosis in the kidney tissue by producing an extracellular matrix. Fibrosis also has a protective role among renal parenchyma diseases including pyelonephritis and defects in renal blood supply. Under stressful conditions, fibrosis could become a scar (hard mass) maintaining some degree of renal structure. Interestingly, scarring also occurs after myocardial infarction in the heart. ${ }^{12}$ Pathological fibrosis is characterized by the deformation of the extracellular matrix and it is classified as sustained, sever, and repetitive. ${ }^{13}$ The developmental stage of fibrosis tissue can mainly be classified into (1) reversible stage and (2) irreversible stage. A number of studies have shown that fibrosis does not stop progressing and it can also be reversible. It was reported that resveratrol inhibits oxidative stress and interstitial fibrosis of kidney in mice models. ${ }^{14}$

\section{TGF- $\beta$ 1 Signaling}

TGF- $\beta 1$ acts as a double-edged sword. It could terminate the inflammatory response and interfere with wound healing, cell differentiation, and cell proliferation, and on the other hand, it may prevent apoptosis in myofibroblasts. TGF- $\beta 1$ increases the number of fibroblasts, converts them to myofibroblasts, and increases the extracellular matrix. Blocking the TGF- $\beta 1$ signaling pathway is an interesting and useful approach for the treatment of renal fibrosis. ${ }^{15}$ When the TGF- $\beta 1$ ligand binds to its receptor, it is activated inside the cell by the transcription factor Smad, through which the p15 gene expression is upregulated, thereby inhibiting CDK4 which results in the arresting cell-cycle at the G1 stage. TGF- $\beta 1$, on the other hand, acts through the Smad and non-Smad pathway. Upon the activation of the Smad pathway, transcription factor $S m a d 2 / 3$ is phosphorylated, which makes complexes with Smad4 and they induce snail gene expression and collagen production and also induces fibrosis. The non-Smad or TAK1 pathway contains PI3K/ AKT, Pho-like GTPase, MAPKsMMK3-P38, and MMK4JNK. TAK1 or TGF- $\beta 1$ activated kinase 1 belongs to the MAPK family, a serine-threonine kinase that is activated by TGF- $\beta 1$ (Figure 1 and 2). The combination of map kinase with TGF- $\beta 1$ signaling pathways induce epithelial cells to undergo changes and become myofibroblasts and it is involved in normal kidney physiology and different types of kidney disease. The kinase is divided into two groups: the first group contains ERK1, 2, 5, p38 kinase alpha, beta, delta, JNK1, 2 and the second group contains ERKs 3, 4, 7, 8. ${ }^{16}$ Map kinases are involved in regulation, proliferation, differentiation, apoptosis, inflammation, and fibrosis. MAP kinases are expressed in all cell types. In fact, the low-level activity of the map kinase is observed in normal human and rodent kidneys. Angiotensin II, IL1, and hypoglycemia may increase the expression of the TGF- $\beta 1$ transcription gene in cases of renal injury. ${ }^{16}$

TAK1 is also activated by environmental stress and proinflammatory cytokines such as TNFa, IL1, and LPS. Smad is a downstream transcription factor of the TGF- $\beta$ 1 receptor. Three types of Smad proteins are active in the TGF- $\beta 1$ signaling pathway: R-Smad (Smad receptorregulated $\operatorname{Smad} 2 / 3)$, co-Smad $(\operatorname{Smad} 4)$, and I-Smad, which inhibit Smad. Regulated Smads have two MH1 and $\mathrm{MH} 2$ domains separated by a flexible interface. The $\mathrm{N}$-terminal region of the second $\mathrm{MH} 1$ has a specific DNA binding fragment as well as a sequence called nuclear localization signal (NLS) nuclear translocation message. NLS sequences are present in all transcription factors in the cytosol and they are necessary for translocation into the nucleus. When the regulatory Smads are inactive, the inactivate phosphorylated form of NLS is coated and the $\mathrm{MH} 1$ and $\mathrm{MH} 2$ domains bind in a manner that cannot be coupled to DNA or co-Smad. Three-fold phosphorylation of the serine cleaves the domains and it binds to importin that can act as an adaptor protein to bind the NLS, allowing Smad to enter into the nucleus, suggesting that TAK1 is activated by AMPK and ischemia agonists, which in turn activates the LKB1/AMPK pathway. TAK1 is also involved in Wnt signaling (Figure 1).

Activation of TGF- $\beta 1$ through the cascade of the MKK3-P38 signaling pathway induces the expression of type I collagen gene in mesangial cells. Through the MKK4-JNK pathway, it may induce the expression of fibronectin. Blocking these two pathways MKK3 


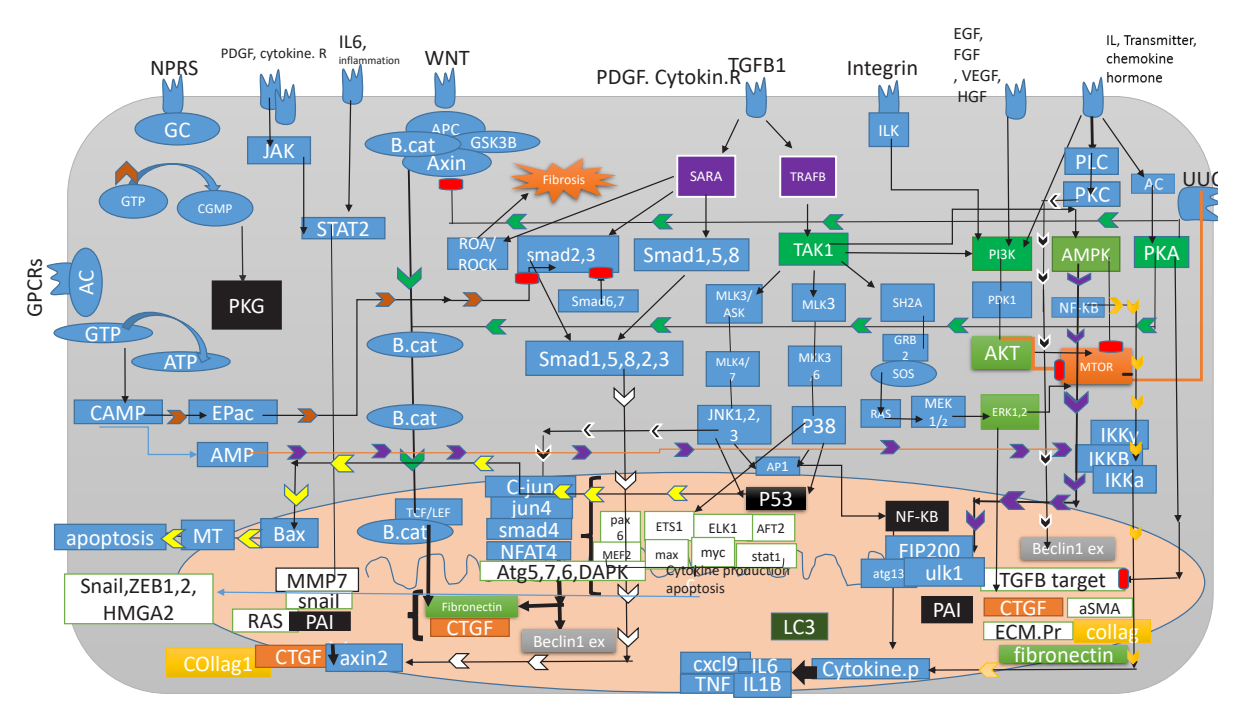

Figure 1. The Map Signaling Pathway in Kidney Fibrosis.

and MKK4 in unilateral urethral obstruction reduces inflammation and fibrosis. In epithelial cells and fibroblasts, TGF- $\beta 1$ expresses extracellular matrix proteins such as collagen and fibronectin and in turn expresses PAI-1 serum protease inhibitor proteins, which inhibits the extracellular matrix and the cell allows to form stable tissue in the cells.

Lack of TGF- $\beta 1$ signaling plays a key role in the spread of many types of cancers. TAB1 (TAK1 Binding protein1) is essential for TAK1 activity. The TAK1-TAB1 complex is essential for fetal development and morphogenesis. ${ }^{10,15}$ On the other hand, in the TAK1 pathway, factors such as angiotensin II, PDGF, and IL1 activate the cascade pathway of JNK, P38, and ERK, which activate pre-fibrotic genes. The aSMA expression in fibroblasts plays a key role in extracellular matrix remodeling which is expressed by TGF- $\beta 1$ signaling. ${ }^{16}$ CTGF, TGF- $\beta 1$, AGE, and IL1

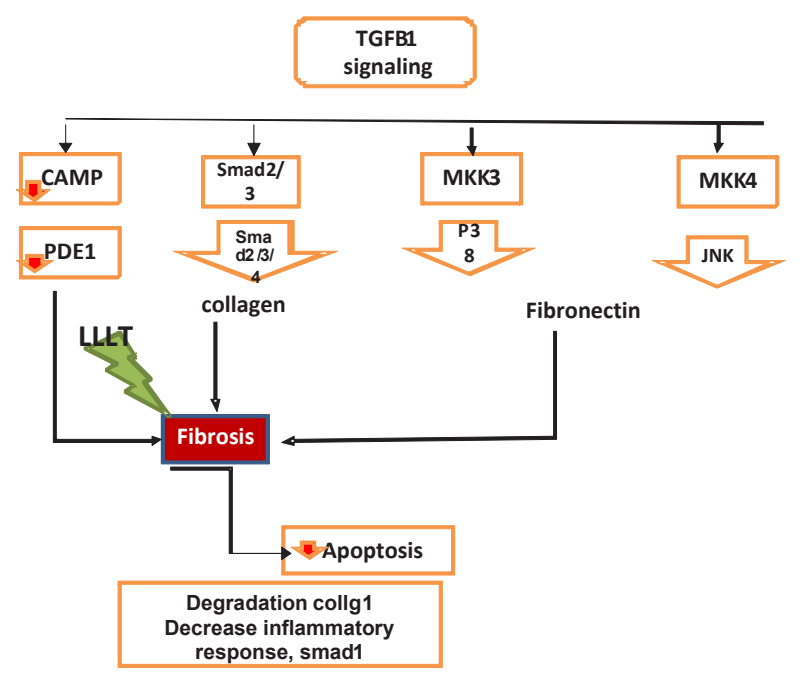

Figure 2. Effect of LLLT on TGFB1 Signaling. cause EMT. In fact, TGF- $\beta 1$ signaling induces EMT that forms the ERK, p38 pathway. The drug sp600125 has been used in mouse keratinocytes, showing that it could suppress TGF- $\beta 1$ in the induction of EMT. During renal fibrosis, the expression of protein genes and mRNAs of extracellular matrix synthesizing proteins increase, which is related to the function of the AP promoter locus at the site of fibronectin and collagen type II genes. ${ }^{16}$ The TGF- $\beta 1$ gene promoter, is a binding site for the AP1 transcription factor. AP1 is a transcription factor that contains both homodimer and heterodimer JUN, FOS, AFT. ERK and JNK play important roles in enhancing c-Jun and $\mathrm{c}$-fos expression and phosphorylation and activation of AP1. ${ }^{16}$ Ang II causes fibrosis through the JNK p38 pathway and hyperglycemia through the ERK, p38 pathway, and IL1 and TNFa through the JNK and ERK pathway. Chronic inflammation also causes fibrosis in the kidney through the p38 and JNK pathways. In the unilateral urethral obstruction model, the application of NPC31169, a p38 inhibitor, reduced the mRNA of type 4 collagen. Reducing renal fibrosis and blocking p38 had no effect on interstitial macrophage filtration. In the chronic allograft nephropathy model, the p38 inhibitor also reduced MCP1 mRNA levels and macrophage filtration. The administration of JNK inhibitors, such as CC401, showed that the drug reduced apoptosis in tubular cells in the obstructive kidney model. The genetic deletion of JNK1/2 does not prevent the spread of renal fibrosis in the unilateral obstructive model but does reduce apoptosis in tubular cells (Figure 2).

The administration of $\mathrm{u} 0126$ as a MEK1 inhibitor showed that although it had a major role in reducing the proliferation and accumulation of interstitial macrophages, it had failed to halt myofibroblasts accumulation and spread fibrosis. Inhibitors of p38 in rheumatoid arthritis and Crohn's disease cause toxicity 
and elevate liver enzymes and cause dizziness. A JNKspecific inhibitor such as CEP1347 inhibits MLK3 and suppresses JNK. ${ }^{16}$

\section{Laser Therapy for Another Kidney Disease}

There are several ways to CKD treatment, such as induction of autophagy, cell therapy and laser therapy. In previous studies, retinoic acid was used for induction of autophagy in stem cells. ${ }^{17}$ It seems that it may be promising for $\mathrm{CKD}$ as well. In addition, differentiating various cell lines may enable cell therapy for various disorders. ${ }^{18}$ Recently Laser therapy is one promising alternative for the treatment of the disease. The low-power laser helps to heal and repair musculoskeletal injuries by reducing inflammation, increasing proangiogenic activity, and increasing the migration and proliferation of epithelial cells. Studies have also focused on repairing the nervous system with the laser (Table 1). Many of the therapeutic potentials of the low-power laser can also be effective in the treatment of renal fibrosis.

In a study, the low-wavelength laser at 635, 532 and 405 $\mathrm{nm}$ was used on renal fibrosis with mesenchymal stem cells in rats. The results showed that the use of the 635 $\mathrm{nm}$ low-power laser increased the mitochondrial activity. The increased proliferation of glomerular cells at $635 \mathrm{~nm}$ with MSC increased IL10 anti-inflammatory cytokines. The TGFB1 fibrosis cytokine was also significantly reduced at $532 \mathrm{~nm}$ with laser mesenchymal stem cells. Apoptosis decreased in all types of wavelengths. The results showed that the laser at 635 and $532 \mathrm{~nm}$ with mesenchymal stem cells would have faster healing activity in renal fibrosis. ${ }^{16}$ Another study also demonstrated that lasers of different wavelengths induce different responses in the kidney cortex. This is an important marker for kidney fibrosis. ${ }^{19}$ It has also been shown that lasers (AlGaAs laser, $780 \mathrm{~nm}, 22.5 \mathrm{~J} / \mathrm{cm}^{2}, 30 \mathrm{~mW}, 0.75 \mathrm{~W} /$ $\mathrm{cm}^{2}, 30$ seconds) have a protective effect on fibrosis and they can reduce transdifferentiation and tubular activity associated with fibrosis by reducing inflammation. After 14 days of urethral obstruction, they reduced IL6 MCP-1, IL6 and decreased fibrotic factors such as TGFB1 and Smad1 $1{ }^{20}$ Diabetes may alter the antioxidant defense system. ${ }^{21}$ Mice with diabetic nephropathy have a high level of oxidative damage to lipids along with high levels of blood urea nitrogen (BUN), leading to impaired kidney function. ${ }^{22}$ Unsaturated fatty acids in the plasma membrane are the main targets of ROS. Structural changes in membrane lipids result in the loss of membrane phospholipids, decreased membrane fluidity, and decreased activity of membrane anchor enzymes and receptors, and they significantly impair performance. ${ }^{22}$ Neutrophils produce very high levels of ROS during acute ischemia. During inflammation, the number of inflammatory cells increases. Myeloperoxidase (MPO) in neutrophils increases during inflammatory responses that catalyze the structure of hypochlorous acid, which is a toxin for the cellular structure. In renal IR injury, ROS interaction with lipids results in the peroxidation of biological membrane lipids. If lipid peroxidation remains unchecked, it will eventually lead to cell death. A lowpower laser treatment $\left(685 \mathrm{~nm}, 15 \mathrm{~mW}, 3.2 \mathrm{~J} / \mathrm{cm}^{2}\right)$ on diabetic rats with right nephrectomy showed a significant decrease in glutathione, and significant improvement in both superoxide dismutase (SOD) and catalyzing levels as well as a significant decrease in the level of MPO and ROS comparing to nephrectomy group..$^{22}$

Metabolic syndrome is characterized by its features of hyperglycemia, hypertension, obesity, and dyslipidemia. Hyperglycemia itself increases ROS in the cell. Chronic diabetes also decreases the structural and functional impairment of renal cell function by decreasing $\mathrm{Na}^{+} / \mathrm{K}^{+}$ATPase activity. Among several active kidney nephron carriers, $\mathrm{Na}^{+} / \mathrm{K}^{+}$-ATPase is the most widespread and most important active transport system that plays an important physiological role in regulating cellular homeostasis. ${ }^{23}$ Diabetes also causes inactivation of antioxidant enzymes such as glutathione peroxidase, glutathione reductase and catalase, SOD, glutathione S-transferase, and glutathione. Antioxidant enzymes act as an antioxidant defense system against tissue damage such as ROS, resulting in inactivation of these enzymes and impaired protein expression. ${ }^{23}$ The low-powered laser $\left(12 \mathrm{~J} / \mathrm{cm}^{2}\right.$, $5 \mathrm{~mW}$; treatment time 20.4) can be effective in rats suffering from nephrectomy, hypertension, dyslipidemia, and type 2 diabetes and can reduce blood pressure and increase glomerular filtration rate, and decrease collagen fiber accumulation. ${ }^{24}$ The low-power laser $\left(670 \mathrm{~nm}, 9 \mathrm{j} / \mathrm{cm}^{2}, 14\right.$ days) increased sodium-potassium activity and catalyzed activity and sodium-potassium ATPase activity in all light-treated diabetic rats, whereas

Table 1. The List of Studies About CKD and LLL

\begin{tabular}{|c|c|c|c|c|c|c|c|}
\hline Author & Wavelength $(\mathrm{nm})$ & $\begin{array}{c}\text { Output } \\
\text { Power }(\mathrm{mW})\end{array}$ & $\begin{array}{l}\text { Laser Beam } \\
\qquad\left(\mathrm{cm}^{2}\right)\end{array}$ & $\begin{array}{l}\text { Energy Density } \\
\qquad\left(\mathrm{J} / \mathrm{cm}^{2}\right)\end{array}$ & $\begin{array}{l}\text { Irradiation } \\
\left(\mathrm{mw} / \mathrm{cm}^{2}\right)\end{array}$ & $\begin{array}{c}\text { Exposure } \\
\text { Duration (s) }\end{array}$ & Model of Disease \\
\hline $\mathrm{O}^{\prime}$ Connor et $\mathrm{al}^{19}$ & 635,532, or 405 & 17.5 & 3 & $3.662 \mathrm{~mJ} / \mathrm{cm}^{2}$ & 12.20 & 300 & Fibrosis kidney \\
\hline Oliveira et $\mathrm{al}^{20}$ & 780 & 30 & $4 \mathrm{~mm}^{2}$ & 22.5 & 0.75 & 30 & Fibrosis kidney \\
\hline Asghari et $\mathrm{al}^{22}$ & 685 & 15 & 0.28 & 3.2 & & 60 & Diabetic \\
\hline Ucero et $\mathrm{al}^{24}$ & 785 & 5 & 1 & 12 & 15 & 20.4 & Metabolic syndrome related kidney injury \\
\hline Lim et $\mathrm{al}^{23}$ & 760 & - & - & 10.5 & 35 & 300 & Diabetic \\
\hline
\end{tabular}


the activity of glutathione peroxides and expression increased and the expression of sodium-potassium ATPase was unchanged. ${ }^{23}$ LLLT decreased serum BUN, serum creatinine and BUN/creatinine ratios and further increased the activity and expression of cytochrome $\mathrm{c}$ oxidase, which is a major photoreceptor molecule in the mitochondrial respiratory chain, and the formation of (8-hydroxy-2'deoxyguanosine) which is a DNA additive that reduces in the kidneys.

Glomerulonephritis, also known as good postural syndrome, is one of the immune system disorders that affect the kidney. The disease produces antibodies against the glomerular basement membrane (GBM) membrane. The disease is often fatal. The accumulation of macrophages and monocytes within the glomeruli and the interstitial space of the tubules give rise to glomerulonephritis, which is the major accumulation of macrophages in the glomeruli. Macrophages produce proinflammatory factors such as tumor necrosis factor alpha (TNF- $\alpha$ ), MMPs12, and ROS that activate the proinflammatory signaling pathway c-Jun, p38, NF-KB. A study showed that low-power lasers (irradiation spot size on skin surface, $2.27 \mathrm{~cm}^{2}$; power intensity, $880 \mathrm{~mW} / \mathrm{cm}^{2}$; irradiation mode, continuous mode; irradiation time, $250 \mathrm{~s}$; energy, $500 \mathrm{~J}$; energy density, $220 \mathrm{~J} / \mathrm{cm}^{2}$ ) could attenuate IL1 $\beta$ and TNF- $\alpha$ levels for 14 days in the cortex of all rats that received intravenous glomerulonephritis antibody against GBM. ${ }^{24}$ Chemical induction can also cause cellular stress and cellular oxidative stress. One use of TCDD (2,3,7,8-tetrachlorodibenzo-p-dioxin) is in the studies that cause oxidative stress and sub-lethal changes. One study used TCDD in all 20-day-old embryos that showed a decrease in the level of ATP, Glutathione, and lipid peroxidation. The low-power laser $\left(670 \mathrm{~nm}, 4 \mathrm{~J} / \mathrm{cm}^{2}\right)$ showed that it could normalize lipid peroxidase levels, an antioxidant enzyme, and glutathione contents based on TCDD changes compared to the control group. ${ }^{25}$

Acute kidney injury is a well-known renal complication caused by many factors such as ischemia, obstructive injury or drug-based toxicity. Cisplatin is an antineoplastic drug used in the treatment of many cancers. Cisplatin-induced cytotoxicity has been known since its introduction more than 25 years ago; cisplatin damages nuclear and mitochondrial DNA and produces ROS that activate mitochondrial and non-mitochondrial pathways. Mitochondria cause cell death through apoptosis and necrosis. Numerous evidence suggests that mitochondrial DNA or other mitochondrial targets play a more important role in inducing cisplatin-induced cell death than nuclear DNA damage. ${ }^{26}$

\section{Conclusion}

It was shown that lasers can be effective in reducing inflammatory responses and fibrosis factors and decreasing ROS levels in kidney disease. It can also enhance anti-inflammatory responses and glomerular cell proliferation.

\section{Ethical Considerations}

This study has been approved by the ethical committee of Shahid Beheshti University of Medical Sciences.

\section{Conflict of Interests}

The authors declare no conflict of interest.

\section{Acknowledgment}

This work was supported by the Laser application in Medical Sciences Research Center of Shahid Beheshti University of Medical Sciences, Tehran, Iran.

\section{References}

1. López-Hernández FJ, López-Novoa JM. Role of TGF- $\beta$ in chronic kidney disease: an integration of tubular, glomerular and vascular effects. Cell Tissue Res. 2012;347(1):141-54. doi: 10.1007/s00441-011-1275-6.

2. Harris RC, Neilson EG. Toward a unified theory of renal progression. Annu Rev Med. 2006;57:365-80. doi: 10.1146/ annurev.med.57.121304.131342.

3. Mutsaers HA, Olinga P. Editorial: Organ fibrosis: triggers, pathways, and cellular plasticity. Front Med (Lausanne). 2016;3:55. doi: 10.3389/fmed.2016.00055.

4. Couser WG, Remuzzi G, Mendis S, Tonelli M. The contribution of chronic kidney disease to the global burden of major noncommunicable diseases. Kidney Int. 2011;80(12):1258-70. doi: 10.1038/ki.2011.368.

5. Jha V, Garcia-Garcia G, Iseki K, Li Z, Naicker S, Plattner $\mathrm{B}$, et al. Chronic kidney disease: global dimension and perspectives. Lancet. 2013;382(9888):260-72. doi: 10.1016/ S0140-6736(13)60687-X.

6. Wynn TA. Cellular and molecular mechanisms of fibrosis. $J$ Pathol. 2008;214(2):199-210. doi: 10.1002/path.2277.

7. Hewitson TD. Renal tubulointerstitial fibrosis: common but never simple. Am J Physiol Ren Physiol. 2009;296(6): F1239-F1244. doi: 10.1152/ajprenal.90521.2008.

8. Dungey M, Hull KL, Smith AC, Burton JO, Bishop NC. Inflammatory factors and exercise in chronic kidney disease. Int J Endocrinol. 2013;2013:569831. doi: 10.1155/2013/569831.

9. Rockey DC, Bell PD, Hill JA. Fibrosis-a common pathway to organ injury and failure. $N$ Engl $\mathrm{J} \mathrm{Med}$. 2015.372(12):1138-1149. doi: 10.1056/NEJMra1300575.

10. Duffield JS. Cellular and molecular mechanisms in kidney fibrosis. J Clin Invest. 2014;124(6): 2299-2306. doi:10.1172/ JCI72267.

11. Herrera J, Henke CA, Bitterman PB. Extracellular matrix as a driver of progressive fibrosis. J Clin Invest. 2018;128(1): 45-53. doi: 10.1172/JCI93557.

12. Bechtel W, McGoohan S, Zeisberg EM, Müller GA, Kalbacher H, Salant DJ, et al. Methylation determines fibroblast activation and fibrogenesis in the kidney. Nat Med. 2010;16(5):544-50. doi: 10.1038/nm.2135.

13. Koyama Y, Xu J, Liu X, Brenner DA. New developments on the treatment of liver fibrosis. Dig Dis. 2016;34(5):589-96. doi: $10.1159 / 000445269$.

14. Koushki M, Amiri-Dashatan N, Ahmadi N, Abbaszadeh 
HA, Rezaei-Tavirani M. Resveratrol: A miraculous natural compound for diseases treatment. Food Sci Nutr. 2018;6(8):2473-90. doi: 10.1002/fsn3.855.

15. Choi ME, Ding Y, Kim SI. TGF- $\beta$ signaling via TAK1 pathway: role in kidney fibrosis. Semin Nephrol. 2012;32(3):244-52. doi: 10.1016/j.semnephrol.2012.04.003.

16. Ma FY, Sachchithananthan M, Flanc RS, Nikolic-Paterson DJ. Mitogen-activated protein kinases in renal fibrosis. Front Biosci (Schol Ed). 2009;1:171-87. doi: 10.2741/s17.

17. Darabi S, Tiraihi T, Noori-Zadeh A, Rajaei F, Darabi L, Abbaszadeh H. Creatine and retinoic acid effects on the induction of autophagy and differentiation of adipose tissue-derived stem cells into GABAergic-like neurons. Journal of Babol University of Medical Sciences. 2017;19(8):41-9. doi: 10.22088/jbums.19.8.41.

18. Abbaszadeh HA, Tiraihi T, Delshad A, Saghedizadeh M, Taheri T, Kazemi H, et al. Differentiation of neurospherederived rat neural stem cells into oligodendrocyte-like cells by repressing PDGF- $\alpha$ and Olig2 with triiodothyronine. Tissue Cell. 2014;46(6):462-9. doi: 10.1016/j. tice.2014.08.003.

19. O'Connor M, Patil R, Yu J, Hickey R, Premanand K, Kajdacsy-Balla A, et al. Mesenchymal stem cells synergize with 635, 532, and $405 \mathrm{~nm}$ laser wavelengths in renal fibrosis: a pilot study. Photomed Laser Surg. 2016;34(11):556-563. doi:10.1089/pho.2015.4025.

20. Oliveira FA, Moraes AC, Paiva AP, Schinzel V, CorreaCosta M, Semedo P, et al. Low-level laser therapy decreases renal interstitial fibrosis. Photomed Laser Surg. 2012; 30(12): 705-13. doi:10.1089/pho.2012.3272.

21. Maritim AC, Sanders RA, Watkins JB 3rd. Diabetes, oxidative stress, and antioxidants: a review. J Biochem $\mathrm{Mol}$ Toxicol. 2003;17(1): 24-38. doi: 10.1002/jbt.10058.

22. Asghari A, Takhtfooladi MA, Hoseinzadeh HA. Effect of photobiomodulation on ischemia/reperfusioninduced renal damage in diabetic rats. Lasers Med Sci. 2016;31(9):1943-1948. doi: 10.1007/s10103-016-2073-x.

23. Lim J, Sanders RA, Snyder AC, Eells JT, Henshel DS, Watkins JB 3rd. Effects of low-level light therapy on the streptozotocin-induced diabetic kidney. J Photochem Photobiol B. 2010;99(2):105-110. doi: 10.1016/j. jphotobiol.2010.03.002.

24. Ucero AC, Sabban B, Benito-Martin A, Carrasco S, Joeken S, Ortiz A. Laser therapy in metabolic syndrome-related kidney injury. Photochem Photobiol. 2013;89(4):953-960. doi: 10.1111/php.12055.

25. Lim J, Sanders RA, Yeager RL, Millsap DS, Watkins JB 3rd, Eells JT, et al. Attenuation of TCDD-induced oxidative stress by $670 \mathrm{~nm}$ photobiomodulation in the developmental chicken kidney. J Biochem Mol Toxicol. 2008;22(4):230-9. doi: 10.1002/jbt.20233.

26. Wada Y, Iyoda M, Matsumoto K, Shindo-Hirai Y, Kuno Y, Yamamoto Y, et al. Epidermal growth factor receptor inhibition with erlotinib partially prevents cisplatin-induced nephrotoxicity in rats. PLoS One. 2014;9(11):e111728. doi: 10.1371/journal.pone.0111728. 